Research Paper

\title{
Identification of MicroRNAs Involved in Hypoxia- and Serum Deprivation- Induced Apoptosis in Mesenchymal Stem Cells
}

\author{
Yu Nie, Bian-Mei Han, Xue-Bin Liu*, Jin-Jing Yang, Fang Wang, Xiang-Feng Cong ${ }^{\bowtie}$, Xi Chen $^{\bowtie}$ \\ Research Center for Cardiovascular Regenerative Medicine, Cardiovascular Institute and Fuwai Hospital, Chinese Academy \\ of Medical Sciences and Peking Union Medical College, Beijing, P. R. China \\ * This author now works in Department of Neural Stem Cell Transplantation, Armed Police General Hospital, Beijing, China.
}

$\triangle$ Corresponding author: Xi Chen \& Xiang-Feng Cong, PhD, Research Center for Cardiovascular Regenerative Medicine, Fuwai Hospital, 167 Beishilu Street, Beijing, 100037, China. Tel: +86 010 88398584; Fax: +86 010 88398584; Email: chenxipumc@yahoo.com.cn \& xiangfeng_cong@yahoo.com.cn

(c) Ivyspring International Publisher. This is an open-access article distributed under the terms of the Creative Commons License (http://creativecommons.org/ licenses/by-nc-nd/3.0/). Reproduction is permitted for personal, noncommercial use, provided that the article is in whole, unmodified, and properly cited.

Received: 2011.02.09; Accepted: 2011.06.01; Published: 2011.06.09

\begin{abstract}
The use of bone marrow mesenchymal stem cell- (MSC) transplantation therapy for cardiac diseases is limited due to poor survival of implanted cells. MicroRNAs (miRNAs) have been reported to be involved in regulating almost all cellular processes, including apoptosis. In this study, we found that the miRNA profile was altered during apoptosis induced by hypoxia and serum deprivation (hypoxia/SD). We further revealed that over-expression of miR-21, miR-23a and miR-210 could promote the survival of MSCs exposed to hypoxia/SD. In contrast, down-regulation of miR-21, miR-23a and miR-503 aggravated apoptosis of MSCs. It was indicated that these miRNAs may play important roles during MSC apoptosis induced by hypoxia/SD.
\end{abstract}

Key words: Mesenchymal stem cells, MicroRNA, Hypoxia/serum deprivation, Apoptosis

\section{Introduction}

Bone marrow mesenchymal stem cells (MSCs) have been recognized as potential candidates for use in stem cell therapy to ameliorate cardiac function in cardiac diseases such as myocardial infarction through multiple mechanisms [1,2]. However, MSC-based therapy has the vital limitation of poor viability of MSCs after cell transplantation. For instance, only $1 \%$ or less of MSCs injected into the left ventricle of CB17 SCID/beige adult mice survived within 4 days of injection [3], implying that ischemic microenvironment of the infracted myocardium could not be conducive of MSCs survival. Our previous studies have demonstrated that MSCs undergo apoptosis induced by hypoxia and serum deprivation (hypoxia/SD) in vitro, conditions designed to mimic ischemic myocardium $[4,5]$. Thus, identifying the fac- tors relative to MSC apoptosis and promoting the survival of transplanted MSCs may be vital for successful utilization in cell therapy.

As a species of single-stranded small non-coding RNA, microRNAs (miRNAs) work by annealing to complementary sites in the 3'UTR of target mRNA, thereby preventing translation of the transcript or accelerating its breakdown [6-8]. According to miR-Base, release 16.0 (Sanger), more than 1000 unique miRNAs are encoded in the human genome, and they are predicted to regulate expression of a large part of the human protein-encoding transcriptome. Almost all cell biological processes, including cellular proliferation, differentiation, apoptosis and tumorigenesis, are regulated accurately by miRNAs [9-11]. Nevertheless, the knowledge of the miRNAs involved in MSC 
apoptosis induced by hypoxia/SD is limited. Therefore, exploring the role of miRNAs regulated by hypoxia/SD in MSCs may be helpful to find novel targets to augment the survival of cells after transplantation.

In this study, we have found that, compared with MSCs in normal culture, 60 miRNAs were significantly differentially expressed in hypoxia/SD-treated MSCs; of these, 57 miRNAs were upregulated, and 3 were down-regulated. Over-expression and knockdown approaches were used to investigate the effects of selected miRNAs during MSC exposure to hypoxia/SD.

\section{Materials and Methods}

\section{Rat bone marrow mesenchymal stem cell cul- ture, treatment and transfection}

MSCs were isolated from Sprague-Dawley rats (Vital River Laboratory Animal Inc., Beijing, China), and apoptosis was induced by hypoxia/SD as previously described $[4,5]$. All cells used in the assay were at passages 2 to 3 . All procedures in the present study were approved by the Animal Care Committee of Cardiovascular Institute and Fuwai Hospital.

MicroRNAs were over-expressed or selectively knocked down by transfection of miRNA mimics (duplexes, GenePharma, Shanghai, China) or inhibitors (OMe modified, GenePharma) using siPORT TM $\mathrm{Neo}^{\mathrm{TM}}$ Transfection Agent (NeoFx; Ambion, Austin, Texas) according to the manufacturer's instructions and previous description [12].

\section{Global analysis of microRNA expression by Taq- Man Low-Density Arrays}

Total RNA was extracted from cell suspensions of approximately $10^{5}$ MSCs using Trizol (Invitrogen, Carlsbad, CA). To study the global change of miRNA expression levels, the TaqMan® Low-Density Array (TLDA) Rodent MicroRNA Array Set v2.0 (Applied Biosystems, Foster City, CA) was used to detect and quantify mature miRNAs. Methods were as the manufacturer's instructions and previously described [13-15]. RQ value (relative level of miRNA expression) was calculated by the comparative $2^{-\Delta \Delta \mathrm{Ct}} \mathrm{Ct}$ method with U6 snRNA as endogenous controls with RQ Manager 1.2 (Applied Biosystems).

\section{Verification of TLDA Results by Single-Well qRT-PCR}

To verify the accuracy of our TLDA data, we performed single qPCR experiments for representative miRNAs with single-well TaqMan miRNA assays (Applied Biosystems) in accordance with the manufacturer's protocols and as previously described [16].
Comparative qPCR was performed in 6 replicates. The $2^{-\Delta \Delta \mathrm{Ct}}$ method was used to calculate relative changes in miRNA expression. The results were normalized to levels of U6 snRNA.

\section{Measurement of cell apoptosis}

Apoptosis was detected by bivariate flow cytometry (FCM) using a FACScan-LSR equipped with CellQuest (BD, San Jose, CA) software after cells had been dyed with an Annexin V-FITC Apoptosis Detection Kit (Invitrogen) according to the manufacturer's protocols. Chromosomal condensation was estimated using the chromatin dye Hoechst 33342 (Sigma, St. Louis, MO) as previously described [4].

\section{Measurement of mitochondrial membrane po- tential}

The mitochondrial membrane potential of MSCs was monitored using a JC-1 Mitochondrial Membrane Potential Detection Kit (Beyotime Inst), as recommended by the manufacturer and as previously described $[17,18]$. Cells $\left(2 \times 10^{5}\right)$ were incubated with 2 $\mu \mathrm{g} / \mathrm{ml}$ of JC- 1 for $20 \mathrm{~min}$ at $37^{\circ} \mathrm{C}$ before flow analyses. Red and green fluorescence emissions were analyzed after JC-1 staining.

\section{Results and Discussion}

\section{microRNA expression profile was altered during MSC apoptosis}

Our previous studies have verified that hypoxia/SD induces MSC early apoptosis, with a maximal induction at $6 \mathrm{~h}$ [4]. To explore the function of miRNAs in MSC apoptosis, the preferentially expressed miRNAs in normal culture and in $6 \mathrm{~h}$ hypoxia/SD-treated MSCs were measured using the high throughput TLDA platform. We confirmed the effect of hypoxia/SD on the apoptosis in MSCs (Fig. 1A), and RNA was extracted from a parallel experimental culture for TLDA screening. The analysis of TLDAs revealed that 60 miRNAs that were differentially expressed (> 1.9-fold change) in MSCs were exposed to hypoxia/SD for $6 \mathrm{~h}$. Surprisingly, among these 60, 57 were up-regulated while only 3 were down-regulated by hypoxia/SD compare to MSCs in normal culture (Fig. 1B).

We further analyzed the data and identified miRNAs regulated by hypoxia (miR-21, miR-23a, miR-26a, miR-30b, miR-125b-5p, miR-210) [19,20], miRNAs involved in apoptosis (miR-21, miR-34a) [21-23] and cell cycle (miR-503) [24,25], and miRNAs changed in a clustered manner (miR-345-3p, miR-532-5p). As a result of this, 10 miRNAs were selected from the result of miRNA TLDA experiments for verification by single-well TaqMan qRT-PCR. As 
shown in Fig. 1C, miR-21, miR-23a, miR-34a, miR-210 and miR-503 were all increased more than 1.5 times after exposure of MSCs to hypoxia/SD for $6 \mathrm{~h}$. To investigate the dynamic change in expression of these 5 miRNAs during MSC apoptosis, we analyzed the expression of miRNAs following different durations $(0 \mathrm{~h}$ to $12 \mathrm{~h}$ ) of hypoxia/SD treatment by TaqMan qRT-PCR. With increasing duration of hypoxia/SD in MSCs, apoptosis was increased, and the expression of miRNAs was also increasingly altered (Fig. 2). Inter- estingly, the relative expression of miR-23a and miR-503 rose very quickly, and the other 3 miRNAs (miR-21, miR-34a and miR-210) reached a 1.5-fold increase after $3 \mathrm{~h}$ treatment with hypoxia/SD (Fig. 2B). It has been demonstrated that miR-210 is up-regulated after ischemia preconditioning and is involved in augmenting the survival of rat MSCs [12]. Combining these findings with our results indicates that ischemia preconditioning may promote MSC survival via regulation of various miRNAs.

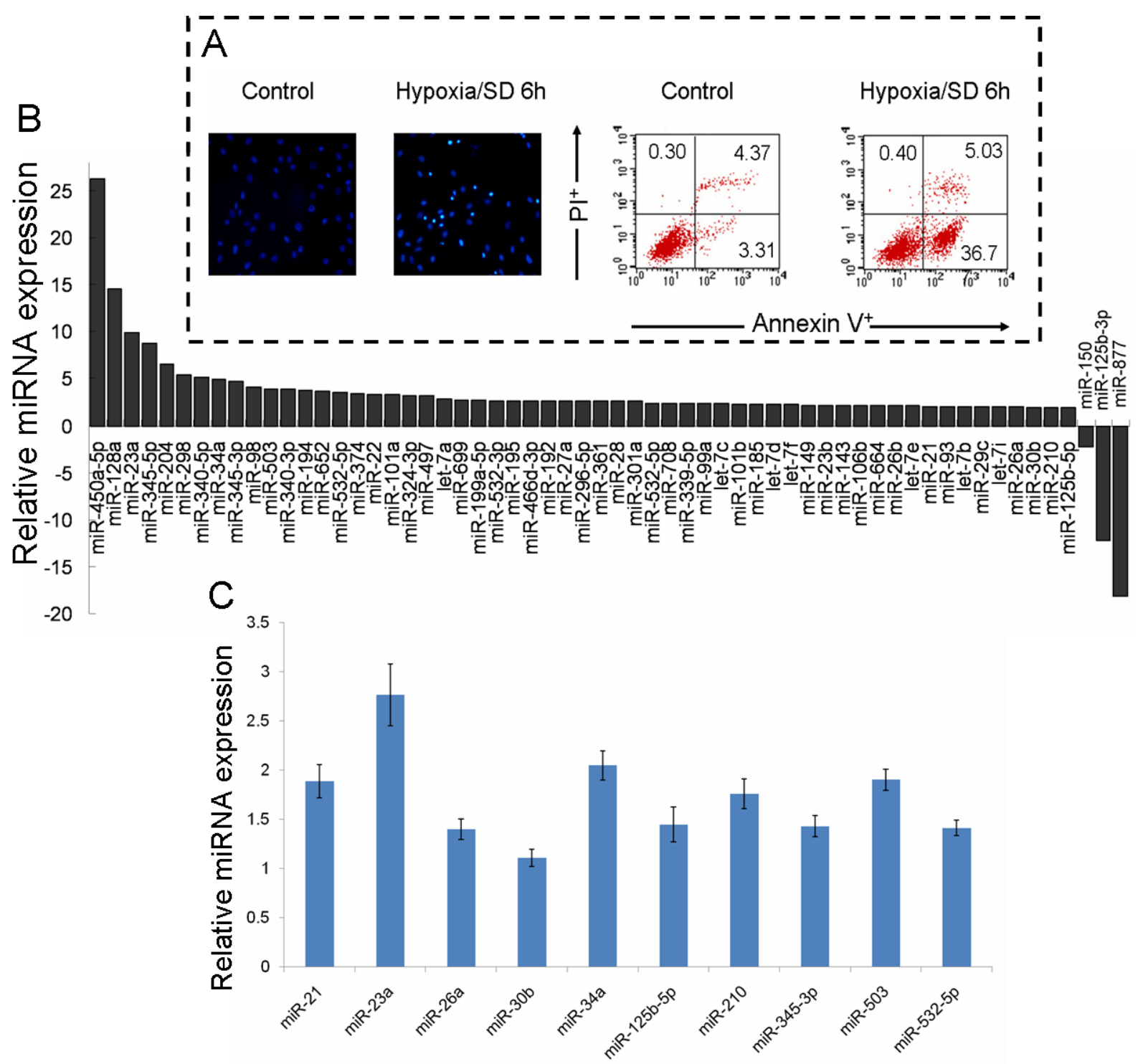

Fig. 1. Multiple miRNAs were regulated during rat mesenchymal stem cell (MSC) apoptosis induced by hypoxia/SD. MSCs were incubated for $6 \mathrm{~h}$ under hypoxia/SD. All experiments were performed in three parallel sets of cultures which were used for different analyses. (A) Apoptosis was identified by detection of cells with apoptotic nuclear morphology using fluorescence microscopy following Hoechst 33342 staining and by fluorescence-activated cell sorting (FACS) analysis after staining with Annexin $\mathrm{V}$ and propidium iodide (PI). (B) Global change of miRNA expression in MSCs was analyzed by using rodent miRNA TLDA screening. (C) Selected miRNAs were verified using single-well TaqMan qRT-PCR. The $2^{-\Delta \Delta C t}$ method was used to calculate relative changes in miRNA expression. The results were normalized to levels of U6 snRNA. Each data point represents mean \pm SEM of six independent experiments. 

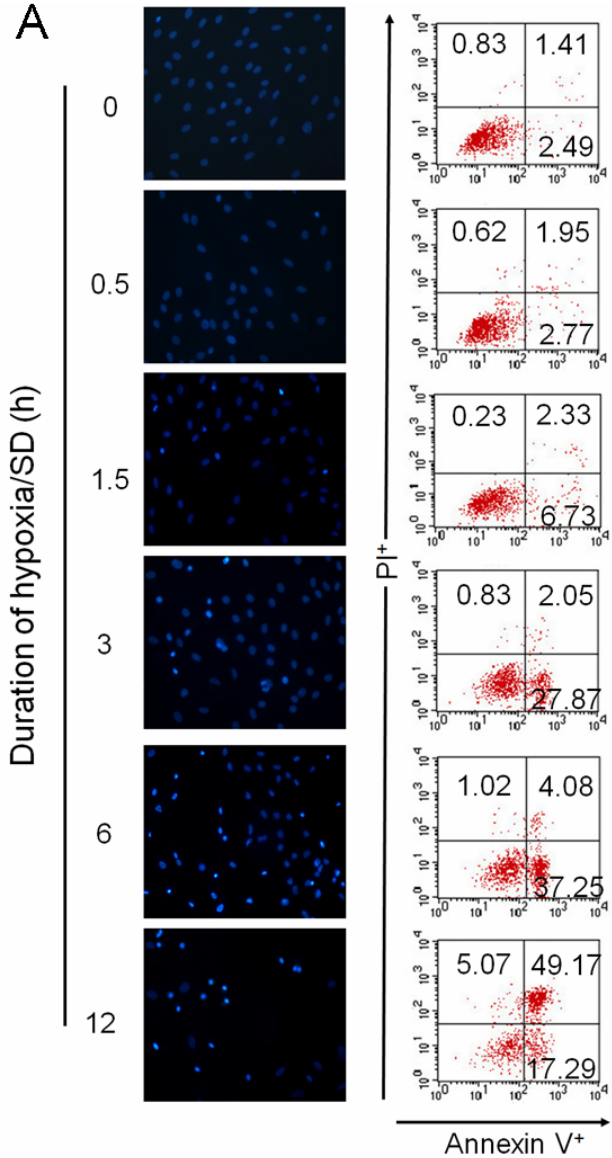

B

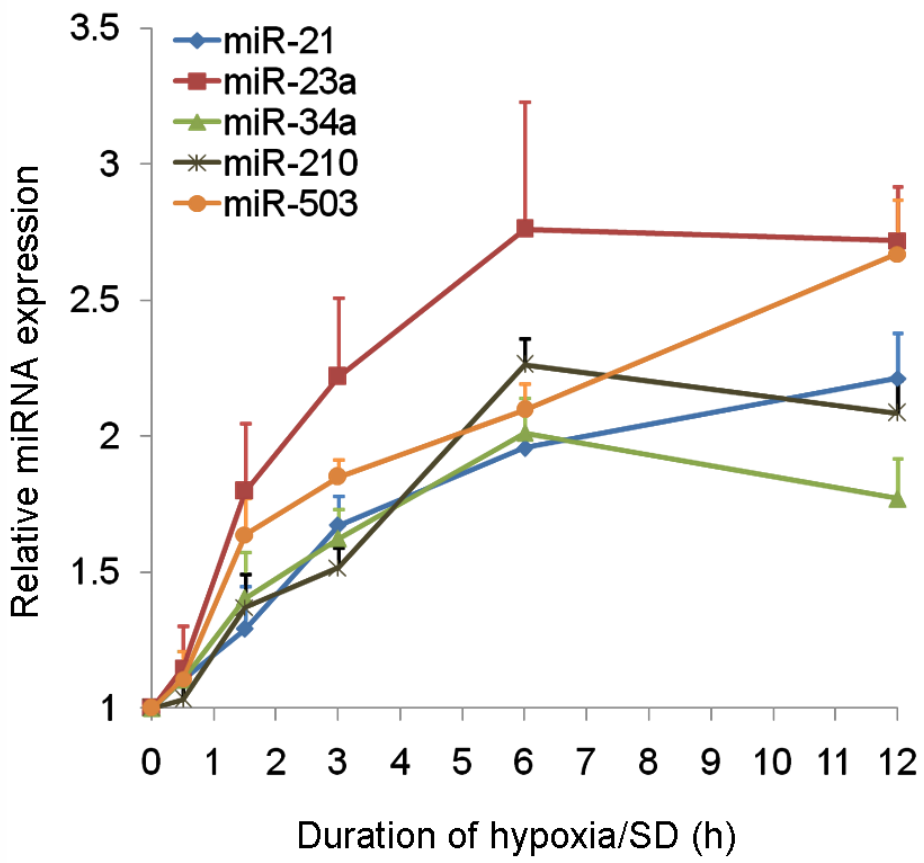

Fig. 2. MicroRNAs were altered dynamically with different duration in hypoxia/SD. The expression of miR-21, miR-23a, miR-34a, miR-210 and miR-503 was dynamically regulated in MSCs under different durations of hypoxia/SD treatment, assessed at $0 \mathrm{~h}, 0.5 \mathrm{~h}, 1.5 \mathrm{~h}, 3 \mathrm{~h}, 6 \mathrm{~h}$ and $12 \mathrm{~h}$. (A) Apoptosis of MSCs at each time-point was determined by Hoechst 33342 staining and by FACS analysis. (B) MicroRNAs were verified by using TaqMan qRT-PCR. Each data point represents mean \pm SEM of six independent experiments.

\section{miRNAs played an important role during MSC apoptosis induced by hypoxia/SD}

To investigate the potential functions of miRNAs in the apoptosis of MSCs, we performed over-expression and knockdown experiments by direct transfection of short double-stranded RNAs (miRNA mimics) or their Ome-modified antisense oligonucleotides (miRNA inhibitors). Effective transfer and blockage of miRNAs were confirmed by TaqMan qRT-PCR (Supplementary Material: Fig. S1). The degree of apoptosis was measured by FCM after cells had been dyed with Annexin V-FITC and propidium iodide (PI).

MiRNAs were specifically suppressed/overexpressed using their corresponding inhibitors/mimics separately in MSCs and then cells were incubated under hypoxia/SD for $6 \mathrm{~h}$ (Fig. 3A). We found that down-regulation of miR-21, miR-23a, or miR-503 precipitated the apoptosis of MSCs. Knockdown of miR-210 increased the amount of MSCs into the later period of apoptosis, but had no effect on the livability of cells (Fig. 3B, 3C). Over-expression of miR-21, miR-23a or miR-210, in contrast, promoted the survival of MSCs when these cells were exposed to hypoxia/SD. We also observed that introduction of miR-503 into MSCs reduced the level of early apoptosis, while increasing the level of later apoptosis, leading to no significant overall change in the total apoptosis level (Fig. 3B, 3D). The involvement in apoptosis/hypoxia of these miRNAs, miR-21, miR-23a and miR-210, has previously been identified in various molecular pathways in different cell types $[12,19,20$, $22,23]$, and miR-503 has been identified as being involved in regulation of the cell cycle [24, 25]. Our findings indicate that these miRNAs may play an 
important role by regulating their target pathways during MSC apoptosis induced by hypoxia/SD. Lossand gain-of-function experiments revealed that
miR-34a had no obvious effect on MSC apoptosis even though they have been previously reported to be involved in apoptosis in other cell types [21].

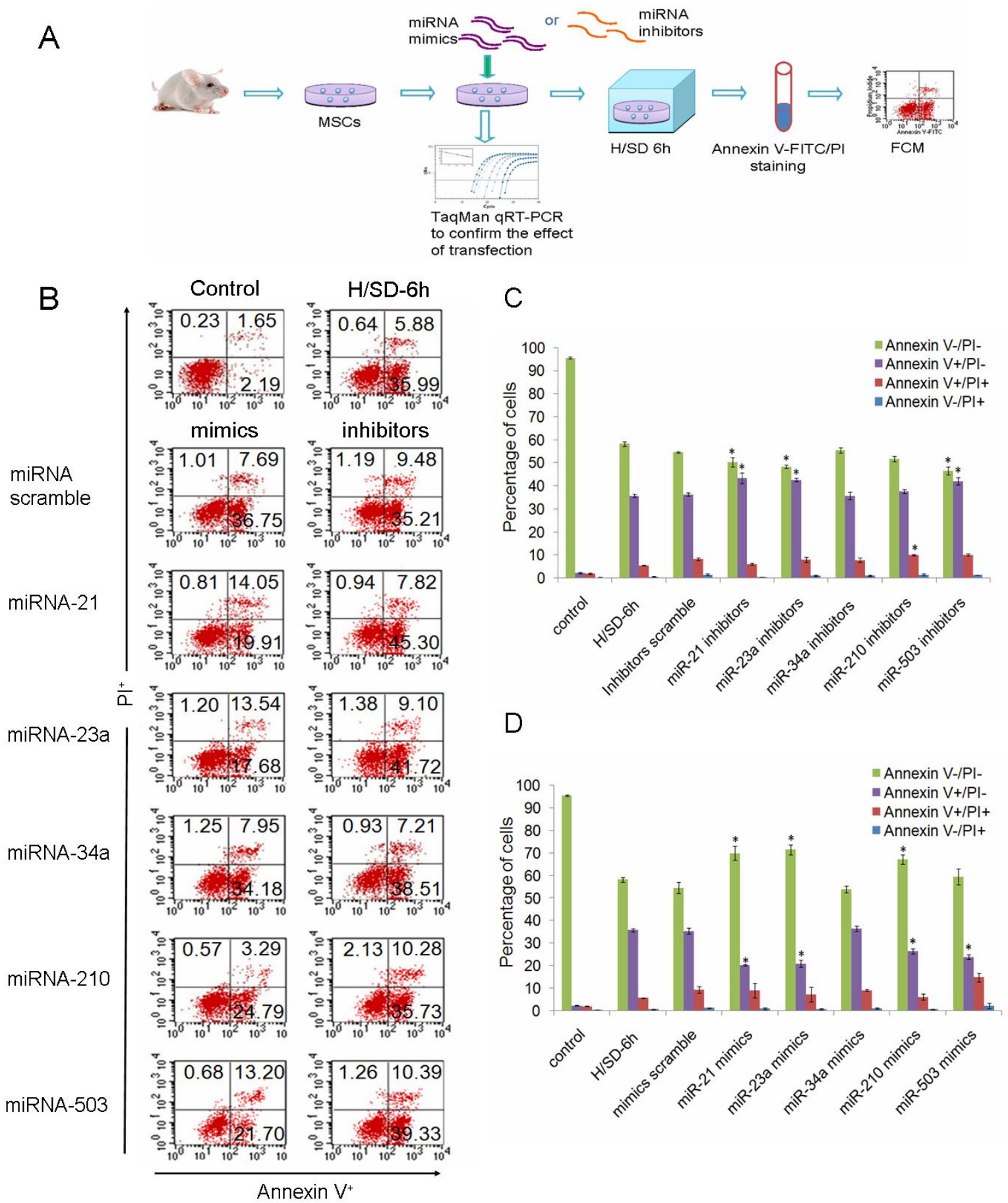

Fig. 3. miR-21, miR-23a, miR-210 and miR-503 were involved in regulation of hypoxia/SD-induced apoptosis in MSCs. (A) MSCs isolated from rats were exposed to hypoxia/SD for $6 \mathrm{~h}$ after transfection with miRNA mimics/inhibitors. Effective transfer and blockage of miRNAs were confirmed by TaqMan qRT-PCR. Apoptosis of MSCs was measured by using FACS analysis after staining with Annexin $\mathrm{V}$ and PI. (B) Viable cells are Annexin $\mathrm{V}^{-} / \mathrm{PI}^{-}$. The Annexin $\mathrm{V}^{+} / \mathrm{PI}^{-}$cells are at an early stage of the apoptotic process, whereas the Annexin $\mathrm{V}^{+} / \mathrm{PI}^{+}$cells have lost cell membrane integrity and thus have taken up PI. Necrotic cells are indicated by Annexin $\mathrm{V}^{-} / \mathrm{PI}^{+}$. (C, D) The results are presented as the percentage of cells. Each data point represents mean \pm SEM of six independent experiments. ${ }^{*} p<0.05$ vs. MSCs transfected with scrambled mimics/inhibitors. 


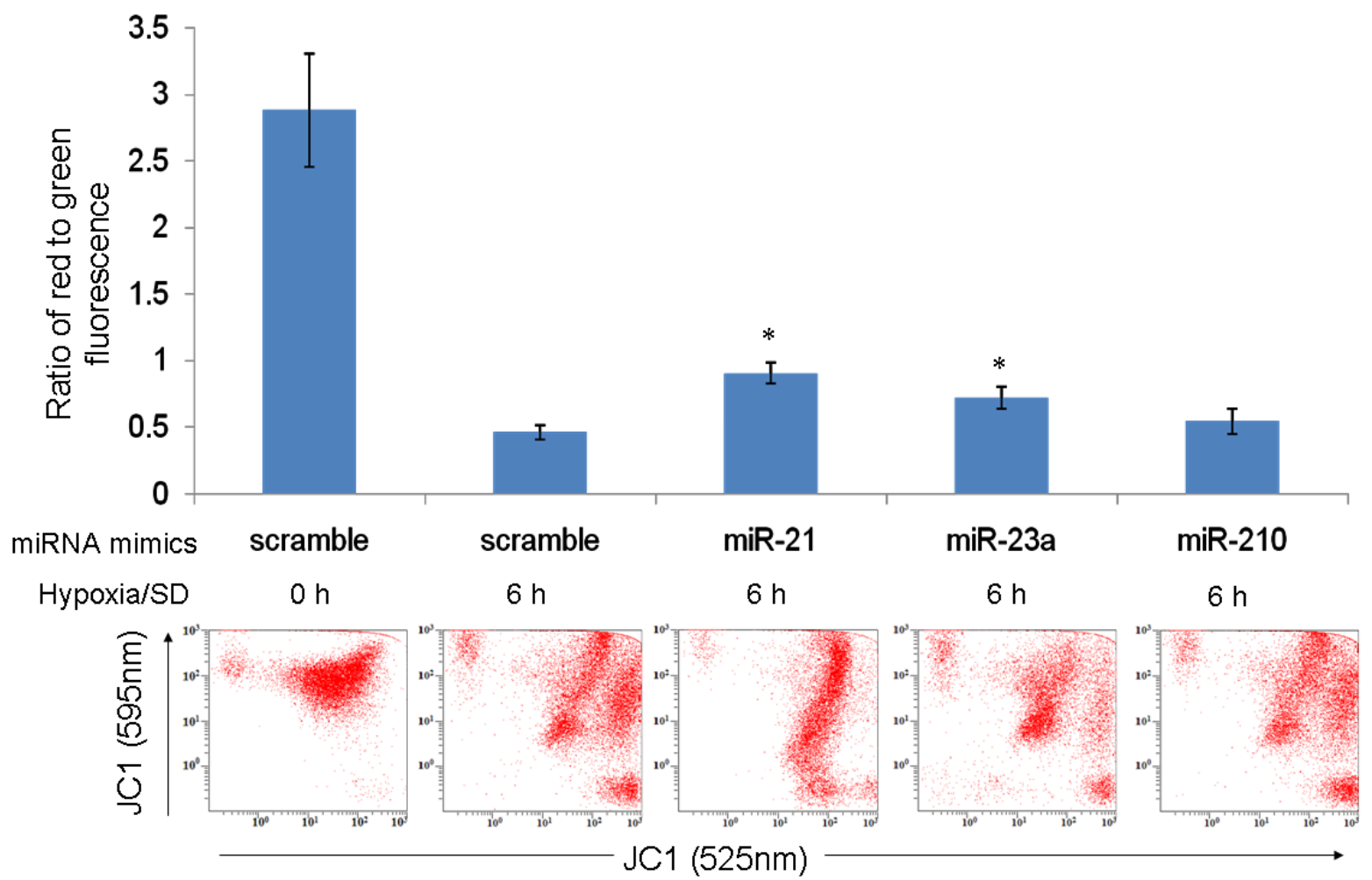

Fig. 4. miR-21 and miR-23a partly prevented the fall in $\Delta \Psi_{m}$ under hypoxia/SD. MSCs transfected with miRNA mimics were treated with hypoxia/SD for $6 \mathrm{~h}$ and mitochondrial potential measured using FACS after JC-1 staining. The ratio of cells with red fluorescence to those with green fluorescence was analyzed. Each data point represents mean \pm SEM of three independent experiments. ${ }^{*} \mathrm{p}<0.05 \mathrm{vs}$. MSCs transfected with scrambled mimics treated with hypoxia/SD $6 \mathrm{~h}$.

The results of our previous investigation showed that hypoxia/SD-induced MSC apoptosis was associated with a loss of mitochondrial membrane potential $(\Delta \Psi \mathrm{m})$ [4]. In the present study, we assessed whether miR-21, miR-23a and miR-210 enhanced mitochondrial function by maintaining $\Delta \Psi \mathrm{m}$. In contrast to MSCs transfected with the mimics scramble group, cells transfected with the mimics of miR-21 and miR-23a partly prevented the fall in $\Delta \Psi \mathrm{m}$ under hypoxia/SD (Fig. 4). This indicated that miR-21 and miR-23a may protect MSCs against hypoxia/SD by preserving mitochondrial function and escaping the mitochondrial apoptotic pathway. MiR-21 has been identified to target programmed cell death 4 (PDCD4), phosphatase and tensin homolog (PTEN) and FasL; all of these pro-apoptotic proteins could trigger the mitochondrial apoptosis pathway $[23,26]$. Fas as the potential target of miR-23a (predicted by using TargetScan algorithms) are also associated with the mitochondrial apoptosis pathway [27]. It's worth noting that miR-210 was unable to rescue the $\Delta \Psi \mathrm{m}$ under hypoxia/SD treatment (Fig. 4). The reason could be because miR-210 over-expression would create mitochondrial dysfunction by targeting the mitochondrial iron-sulfur scaffold protein ISCU [28] as well as members of electron transport chain (ETC) complex: NADH dehydrogenase (ubiquinone) 1 alpha subcomplex, 4 (NDUFA4) and succinate dehydrogenase complex, subunit D (SDHD) [29]. In the meantime, miR-210 could induce a shift to glycolysis [28] and reduce the expression of FLASH/caspase-8 associated protein-2 (FLASH/casp8ap2) [12], thereby enhancing MSC survival without protecting mitochondria.

\section{Summary}

We have demonstrated that the expression of miRNAs is altered by hypoxia/SD in MSCs and that some of these miRNAs play a critival role during MSC apoptosis. Our results indicate that the up-regulation of miR-21, miR-23a and miR-210 induced by hypoxia/SD may be involved in protecting MSCs against apoptosis. Among them, miR-21 and miR-23a augmented MSC survival partly by preventing the fall in $\Delta \Psi$ m under hypoxia/SD; however, miR-210 may resort to other pathways. Interestingly, blockage of miR-21, miR-23a or miR-503 exacerbated apoptosis, 
suggesting that miR-503 might also be one of the hypoxia related miRNAs in MSCs. Exploring the genes and pathways regulated by these miRNAs during apoptosis will lead us to understand the detailed function of these miRNAs in MSCs.

\section{Supplementary Material}

Fig.S1 and Fig.S2

http://www.biolsci.org/v07p0762s1.pdf

\section{Acknowledgments}

The authors are indebted to Dr. Hai-Yong Gu and Jacob Hall Bauer for expert review of the manuscript. This study was supported by the National Natural Science Foundation of China (30770862, 30871024) and the Major National Basic Research Program in the People's Republic of China (Program 973, 2010CB529508, 2007CB512108, 2005CB724600).

\section{Abbreviations}

MSCs: Mesenchymal stem cells; miRNA: microRNA; hypoxia/SD: hypoxia and serum deprivation; TLDA: TaqMan Low-Density Array.

\section{Conflict of Interests}

The authors declare no conflict of interest.

\section{References}

1. Boyle AJ, McNiece IK, and Hare JM. Mesenchymal stem cell therapy for cardiac repair. Methods Mol Biol. 2010; 660: 65-84.

2. Fukuda $\mathrm{K}$, and Yuasa S. Stem cells as a source of regenerative cardiomyocytes. Circ Res. 2006; 98: 1002-13.

3. Geng YJ. Molecular mechanisms for cardiovascular stem cell apoptosis and growth in the hearts with atherosclerotic coronary disease and ischemic heart failure. Ann N Y Acad Sci. 2003; 1010: 687-97.

4. Zhu W, Chen J, Cong X, et al. Hypoxia and serum deprivation-induced apoptosis in mesenchymal stem cells. Stem Cells. 2006; 24: 416-25.

5. Chen J, Baydoun AR, Xu R, et al. Lysophosphatidic acid protects mesenchymal stem cells against hypoxia and serum deprivation-induced apoptosis. Stem Cells. 2008; 26: 135-45.

6. Bartel DP. MicroRNAs: genomics, biogenesis, mechanism, and function. Cell. 2004; 116: 281-97.

7. Wienholds E, and Plasterk RH. MicroRNA function in animal development. FEBS Lett. 2005; 579: 5911-22.

8. Fabian MR, Sonenberg N, and Filipowicz W. Regulation of mRNA translation and stability by microRNAs. Annu Rev Biochem. 2010; 79: 351-79.

9. Inui M, Martello G, and Piccolo S. MicroRNA control of signal transduction. Nat Rev Mol Cell Biol. 2010; 11: 252-63.

10. Krol J, Loedige I, and Filipowicz W. The widespread regulation of microRNA biogenesis, function and decay. Nat Rev Genet. 2010; 11: 597-610.
11. Deng Y, Deng H, Bi F, et al. MicroRNA-137 Targets Carboxyl-terminal Binding Protein 1 in Melanoma Cell Lines. Int J Biol Sci. 2011; 7: 133-7.

12. Kim HW, Haider HK, Jiang $S$, et al. Ischemic preconditioning augments survival of stem cells via miR-210 expression by targeting caspase-8-associated protein 2. J Biol Chem. 2009; 284: 33161-8.

13. Chen Y, Gelfond JA, McManus LM, et al. Reproducibility of quantitative RT-PCR array in miRNA expression profiling and comparison with microarray analysis. BMC Genomics. 2009; 10: 407.

14. Wei H, Wang C, Zhang C, et al. Comparative profiling of microRNA expression between neural stem cells and motor neurons in embryonic spinal cord in rat. Int J Dev Neurosci. 2010; 28: 545-51.

15. Mukhopadhyay P, Mukherjee S, Ahsan K, et al. Restoration of altered microRNA expression in the ischemic heart with resveratrol. PLoS One. 2010; 5: e15705.

16. Chen C, Ridzon DA, Broomer AJ, et al. Real-time quantification of microRNAs by stem-loop RT-PCR. Nucleic Acids Res. 2005; 33: e179.

17. Wei $\mathrm{H}, \mathrm{Li} \mathrm{Z}, \mathrm{Hu} \mathrm{S}$, et al. Apoptosis of mesenchymal stem cells induced by hydrogen peroxide concerns both endoplasmic reticulum stress and mitochondrial death pathway through regulation of caspases, p38 and jnk. J Cell Biochem. 2010; 111: 967-78.

18. Sampson SR, Bucris E, Horovitz-Fried M, et al. Insulin increases h2o2-induced pancreatic beta cell death. Apoptosis. 2010; 15: 1165-76.

19. Kulshreshtha R, Ferracin M, Wojcik SE, et al. A microRNA signature of hypoxia. Mol Cell Biol. 2007; 27: 1859-67.

20. Kulshreshtha R, Davuluri RV, Calin GA, et al. A microRNA component of the hypoxic response. Cell Death Differ. 2008; 15: 667-71.

21. Chang TC, Wentzel EA, Kent OA, et al. Transactivation of miR-34a by 553 broadly influences gene expression and promotes apoptosis. Mol Cell. 2007; 26: 745-52.

22. Krichevsky AM, and Gabriely G. miR-21: a small multi-faceted RNA. J Cell Mol Med. 2009; 13: 39-53.

23. Cheng $\mathrm{Y}, \mathrm{Zhu} \mathrm{P}$, Yang $\mathrm{J}$, et al. Ischaemic preconditioning-regulated miR-21 protects heart against ischae$\mathrm{mia} /$ reperfusion injury via anti-apoptosis through its target PDCD4. Cardiovasc Res. 2010; 87: 431-9.

24. Jiang Q, Feng MG, and Mo YY. Systematic validation of predicted microRNAs for cyclin D1. BMC Cancer. 2009; 9: 194.

25. Sarkar S, Dey BK, and Dutta A. MiR-322/424 and -503 are induced during muscle differentiation and promote cell cycle quiescence and differentiation by down-regulation of Cdc25A. Mol Biol Cell. 2010; 21: 2138-49.

26. Sayed D, He M, Hong C, et al. Microrna-21 is a downstream effector of akt that mediates its antiapoptotic effects via suppression of fas ligand. J Biol Chem. 2010; 285: 20281-90.

27. Thress K, Kornbluth S, and Smith JJ. Mitochondria at the crossroad of apoptotic cell death. J Bioenerg Biomembr. 1999; 31: 321-6.

28. Favaro E, Ramachandran A, McCormick R, et al. Microrna-210 regulates mitochondrial free radical response to hypoxia and krebs cycle in cancer cells by targeting iron sulfur cluster protein iscu. PLoS One. 2010; 5: e10345.

29. Puissegur MP, Mazure NM, Bertero T, et al. Mir-210 is overexpressed in late stages of lung cancer and mediates mitochondrial alterations associated with modulation of hif-1 activity. Cell Death Differ. 2011; 18: 465-78. 